\title{
Proactive Price Regulation for Upgrading Telecommunications Infrastructure
}

\author{
Qiong Wang' and Jon M. Peha ${ }^{2}$ \\ Department of Engineering and Public Policy \\ Carnegie Mellon University
}

\begin{abstract}
The extent to which society benefits from an upgrade to a telecommunications network infrastructure depends on when that upgrade occurs. This paper discusses a proactive pricing approach in which the regulator defines a pricing policy to induce a profit-seeking monopoly carrier to upgrade the infrastructure at the socially desirable time. We discuss how the regulator can determine the optimal time to upgrade the infrastructure, and specify a proactive pricing policy to induce that timing. We also demonstrate a tradeoff between promoting social efficiency and protecting consumer interests in applying that approach.
\end{abstract}

Key Words: Pricing, Infrastructure, Regulation, Telecommunications, Monopoly

Information Economics and Policy, Vol. 9, No. 2, June 1997, pp. 161-76

\footnotetext{
${ }^{1}$ Ph.D. candidate in Engineering and Public Policy. Mail: Department of EPP, Carnegie Mellon University, Pittsburgh, PA 15213. Tel: (412) 268-5617, Fax: (412) 268-3757, e-mail: qw22 @andrew.cmu.edu, homepage: http://www.epp.cmu.edu/ qw22/home.html.

${ }^{2}$ Associate Professor in the Department of Electrical and Computer Engineering and the Department of Engineering and Public Policy, Carnegie-Mellon University. Mail: Department of ECE, Carnegie Mellon University, Pittsburgh, PA 15213. Tel: (412) 268-7126, Fax: (412) 268-2860, e-mail: peha@ece.cmu.edu, homepage: http://www.ece.cmu.edu/ peha.
} 


\section{Section 1}

\section{Introduction}

The rapid progress of today's communication technology provides opportunities for a local telephone company to provide a variety of new features. These features either improve the quality of existing services, or provide consumers with new capabilities. In both cases, the new features can be provided in two phases. In the first phase, the company supplements existing network infrastructure as needed with extra equipment for those customers who are willing to pay for the new feature. This often results in a high cost per customer, and limits the accessibility of the feature to customers who are willing to pay a high price for it. In the second phase, the company makes a large investment to upgrade network infrastructure with advanced technology, so that everyone is able to enjoy the new feature as a part of basic service at a lower cost per customer.

For example, a local telephone company can provide automatic rerouting service to enhance the reliability of its basic service. In phase 1, the company leases redundant transmission terminals to individual subscribers as backup. These redundant terminals are connected through a secondary path that is disjoint from the one in use. If there is a cable cut, or an equipment failure in the central office, subscribers who leased the redundant systems can resume their communications along the backup route (Wrobel, 1990). Obviously, this arrangement is not affordable to most people. However, the automatic rerouting service can become available to everyone if the company moves into phase 2 by upgrading the existing network infrastructure to a Synchronous Optical Network (SONET) Ring. In this case, current transmission systems are replaced by high capacity SONET Add and Drop Multiplexers and telephone central offices are connected into a ring topology. Offices can communicate with one another along both clockwise and counter-clockwise directions on the ring. Consequently, any single failure in transmission systems can be circumvented and no one will be affected ( $\mathrm{Wu}, 1992)$. This two-phased process can also be found in the provision of Integrated Services Digital Network (ISDN) service. In phase 1, the service can be offered through Centrex stations, which is not an option for most consumers. By replacing analog lines and switches with digital ones, a phone company can move into phase 2 , and make the service available to everyone on the network in a costeffective way. Similar cases are likely in the future provision of broadband services, in which beginning phase 2 of the infrastructure upgrade means migrating from a copper network to an all-fiber network, or replacing circuit switching with Asynchronous Transfer Mode (ATM) cell switching.

In all of these cases, one must determine whether to move from phase 1 to phase 2 , and if so, when. The two principal factors in this decision are demand and cost. We consider those cases where demand for the new 
service is increasing over time. In addition, since the technology for phase 2 is relatively new, we assume that its cost is generally decreasing, as opposed to the time-invariant cost of using more mature technology in phase 1 .

Given these trends, when should the infrastructure be upgraded? Obviously, when demand grows sufficiently high, and the cost of beginning phase 2 is low, then phase 2 is preferable. Delaying the infrastructure upgrade will be inefficient to satisfy consumer needs. However, upgrading too early is also detrimental to society because capital is tied up for a longer period of time without generating much social benefit, and future cost reductions cannot be exploited. Thus, the timing of beginning phase 2 should be controlled to maximize the value of the new service to society. From a regulator's perspective, the benefit to society can be measured by multiple criteria (Mitchell and Vogelsang, 1992). Social efficiency and consumer benefit are two important measures we address in this paper. If the regulator is only interested in the former, she should try to maximize social welfare, which is defined as profit plus consumer surplus. If she is only concerned about the latter, then she should try to maximize consumer surplus. Most regulators will set their goals in the broad spectrum between these two extremes. Therefore we use weighted social welfare as the regulator's objective in this paper, which is defined as:

weighted social welfare $=$ consumer surplus $+\alpha *$ profit $; \quad 0 \leq \alpha \leq 1$

A large $\alpha$ indicates emphasis on social welfare while a small $\alpha$ indicates emphasis on consumer surplus.

Although the telephone company decides when to upgrade the infrastructure, the regulator can use price regulation to influence the company's timing. In this paper, we will consider a proactive approach in which the regulator periodically gathers demand and cost information, estimates the optimal upgrade time, and declares in advance the rate increase that the company would be allowed after it improves its basic service by moving from phase 1 to phase 2. With such advance planning, the company can better manage the upgrade process. Moreover, we will show that by using this proactive pricing approach, the regulator can motivate a profit-seeking monopoly to upgrade its infrastructure at the optimal time. This is not generally possible with the commonly used price regulations: rate of return and price cap. Under rate of return regulation, a company is guaranteed a given rate of return on its capital investment, and under price cap regulation, the regulator imposes an overall weighted ceiling on a basket of services and adjusts this ceiling periodically to make it roughly equal to cost (Johnson, 1989; Noll, 1989). Neither approach relates the company's profit with when the infrastructure is upgraded, so neither can induce an optimal timing decision.

We will show how the regulator can determine the upgrade time that maximizes weighted social welfare, and develop proactive pricing policy to induce that timing. In the next section, we formulate the regulator's 
decision as an optimization problem. In section 3, we present general properties of the optimal timing for the company to upgrade the infrastructure, and in section 4 , we discuss proactive pricing policies for inducing that timing. We present our conclusions in section 5.

\section{Section 2}

\section{Problem Formulation}

In this section, we formulate a mathematical model to characterize the regulator's problem of inducing the optimal timing. In $\S 2.1$, we define demand and cost parameters, and in $§ 2.2$ we formulate the mathematical model.

\section{§ 2.1 Demand and Cost Parameters}

The demand for the new feature can be characterized by two functions: the demand function, which describes consumers' willingness to pay for the service, and the diffusion function, which depicts the speed of adopting the new feature by potential buyers. The two functions are characterized as follows:

The demand function $D(\phi, t)$ is defined as the total number of consumers who are willing to pay at least $\phi$ per unit of time for the new feature at time $t . \frac{\partial D(\phi, t)}{\partial \phi} \leq 0$ for any $t$, i.e. a higher price leads to reduced sales. We assume $\frac{\partial D(\phi, t)}{\partial t} \geq 0$ for any $\phi \geq 0$ to reflect the increasing demand for the new feature. We define $t=0$ as the time when the regulator specifies her pricing policy. $D(\phi, t)$ represents past demand if $t<0$, and represents future demand if $t>0 . \quad 0 \leq \phi<+\infty$ and $-\infty<t<+\infty, D(\phi,-\infty)=0$.

However, there could be a delay from the time when a consumer considers the new feature to be worthwhile (i.e. the consumer's willingness to pay exceeds the price) to the time when she actually buys that feature (Bass, 1969). This delay is caused by factors other than the price, such as the hassle cost, or the learning cost. The duration of this delay varies among consumers, ranging from 0 (meaning the subscription is made as soon as willingness to pay exceeds price) to $+\infty$ (meaning the subscription is never made). The delay can be described by a random variable with a Cumulative Distribution Function $F(\tau)$. In marketing literature, $F(\tau)$ is defined as the diffusion function (Bass, 1969), which stands for the probability that a consumer will delay no more than $\tau$ units of time to subscribe to the new feature.

Let $X(\phi, t)$ be the number of adopters at time $t$, given price $\phi$. In phase 1, the provision of the new feature is separated from the provision of the basic service, so that the number of consumers who want to subscribe to the new feature equals $D(\phi, t)$, i.e. the number of consumers whose willingness to pay is no less than the price. In the 
presence of the diffusion effect, not all of these consumers will subscribe to the new feature immediately. The value of $X(\phi, t)$ is determined from the demand function $D(\phi, t)$ and the diffusion function $F(\tau)$ as follows:

$$
X(\phi, t)=\int_{-\infty}^{t} \frac{\partial D(\phi, \mu)}{\partial \mu} F(t-\mu) d \mu
$$

which is the number of consumers who decide to buy the new feature during the period $[\mu, \mu+d \mu)$ and delayed no more than $t-\mu$ units of time to make the purchases, accumulated over all $\mu$ before $t$.

In phase 2 , the new feature is bundled with the basic service. Therefore a consumer either adopts the new feature or has to give up the basic service. Since demand for basic service is highly inelastic, we assume no one will choose the second option, so $X(\phi, t)=D(0, t)$.

The cost of providing the new feature differs from phase 1 to phase 2 . In phase 1 , the new feature is offered by providing individuals with dedicated facilities, so the cost incurred at any given time is proportional to the number of users. Since phase 1 technology is mature, the cost per customer is likely to be stable over time, and we define it as the marginal cost of providing the new feature, noted by a constant $k_{1}$. In phase 2 , by changing the infrastructure, the new feature is made available to every consumer. The major cost is the capital investment to upgrade the infrastructure, which is not sensitive to the number of users. Considering that the on-going research efforts and standardization will reduce required investment over time, we define this fixed cost investment as a function of the upgrade time, noted as $C(t)$, and assume $\frac{d C(t)}{d t} \leq 0$. It is generally true for a new technology that the initial advancement is great, and then the pace of progress slows as further improvements become harder to make, i.e. $\frac{d C^{2}(t)}{d t^{2}} \geq 0$. 


\section{\$2.2 The Mathematical Model}

As mentioned earlier, we consider cases in which the company controls the time to upgrade the infrastructure. The regulator cannot make the timing decision, but controls both the subscription fee of using the new feature in phase 1 , and the allowed rate increase in phase 2 . These pricing decisions can be made to induce the company to upgrade the infrastructure at the socially desirable time, subject to the constraint that the company should at least break-even in each phase.

Let $p$ be the subscription fee specified in phase 1 by the regulator. It is a well established result that to maximize social welfare, or to maximize consumer surplus subject to a break-even constraint, the subscription fee should be set at the marginal cost, i.e. $p=k_{1}$.

The regulator gives the company a rate increase after it upgrades infrastructure so that the company can recover the investment cost, and be given an incentive to begin phase 2 at the time that benefits the society the most. Define $r(t, T)$ to be the increase in total revenue per unit of time resulting from the rate increase in phase 2, where $T$ is the socially desirable upgrade time, and $t$ is the actual upgrade time. The company's profit from upgrading the infrastructure at time $t$ will be $\int_{t}^{+\infty} r(t, T) e^{-\sigma \tau} d \tau-C(t) e^{-\sigma t}=\left[\frac{r(t, T)}{\sigma}-C(t)\right] e^{-\sigma t}$, which is the Net Present Value (NPV) of total revenue increase minus the NPV of the investment cost. To induce the company to begin phase 2 at the socially desirable time (i.e. to let $t=T$ ), $r(t, T)$ should satisfy the following two conditions.

1) the company should be able to at least break-even if it upgrades the infrastructure at $T$ :

$$
\left[\frac{r(T, T)}{\sigma}-C(T)\right] e^{-\sigma T} \geq 0
$$

2) the company's profit is maximized if it choose to upgrade the network at $T$ :

$$
\left[\frac{r(T, T)}{\sigma}-C(T)\right] e^{-\sigma T}>\left[\frac{r(t, T)}{\sigma}-C(t)\right] e^{-\sigma t} \quad \text { for any } t \neq T
$$

For any $r(t, T)$ that satisfies both Equations 2 and 3, define $R(T)=r(T, T) . R(T)$ is the total revenue increase the company gets per unit of time if it upgrades the infrastructure at the regulator's desired time. $R(T)$ is a function of $T$, which means the company's profit depends on when the regulator wants the infrastructure to be upgraded.

Given the demand and cost parameters and constraints defined above, how is the socially desirable time to upgrade the network infrastructure decided? How should the regulator determine a pricing policy to induce the optimal timing? What tradeoffs are there in choosing different proactive pricing policies? In the following, we 
develop a mathematical model to address these issues.

We assume the regulator wants to maximize the NPV of weighted social welfare, which is the sum of the NPV of consumer surplus accumulated in both phases and the total profit multiplied by the regulator's preference parameter $\alpha$. Consumer surplus equals the total willingness to pay of all users minus the total payment to the company. In phase 1 , it is $\int_{k_{1}}^{+\infty} X(\phi, t) d \phi$, and in phase 2 , it is $\int_{0}^{+\infty} D(\phi, t) d \phi-R$. The total profit is the same as that given in Equation 2. The mathematical expression for the regulator's objective function is then:

$$
\Pi(T)=\int_{0}^{T} \int_{k_{1}}^{+\infty} X(\phi, t) d \phi e^{-\sigma t} d t+\int_{T}^{+\infty}\left[\int_{0}^{+\infty} D(\phi, t) d \phi-R(T)\right] e^{-\sigma t} d t+\alpha\left[\int_{T}^{+\infty} R(T) e^{-\sigma t} d t-C(T) e^{-\sigma T}\right]
$$

where the definition of variables and their constraints can be found in Table 1. In Equation 4, $T=0$ means the infrastructure should be upgraded immediately, and $T=+\infty$ means the infrastructure should never be upgraded. In the following analysis, we will ignore these two trivial cases.

[Insert Table 1 here]

\section{Section 3}

\section{Discussions on the Optimal Timing}

Based on the mathematical model developed above, in this section, we study the optimal timing to upgrade the network infrastructure. In $\S 3.1$, we derive the necessary condition for achieving the optimal timing and explain its intuition. In $\S 3.2$ we discuss the impact of input parameters.

\section{\$3.1 The Necessary Condition for the Optimal Timing}

Theorem 1 (see Appendix for proof):

The optimal timing for upgrading the infrastructure, $T^{*}$, must satisfy the following condition:

$$
\int_{0}^{+\infty} D\left(\phi, T^{*}\right) d \phi-\int_{k_{1}}^{+\infty} X\left(\phi, T^{*}\right) d \phi=\alpha\left[\sigma C\left(T^{*}\right)-C^{\prime}\left(T^{*}\right)\right]+(1-\alpha)\left[R\left(T^{*}\right)-\frac{R^{\prime}\left(T^{*}\right)}{\sigma}\right]
$$

The intuition behind Equation 5 can be explained as follows. At the optimal time to begin phase 2, the incremental benefit equals the incremental cost. The incremental benefit is the total value of the new feature to all subscribers if phase 2 begins minus the total value of the new feature to existing users if phase 1 continues. Since the value of the new feature is expressed in the form of consumer willingness to pay, and marginal cost pricing is used in the first phase, the NPV of this incremental benefit can then be expressed as 
$\left[\int_{0}^{+\infty} D\left(\phi, T^{*}\right) d \phi-\int_{k_{1}}^{+\infty} X\left(\phi, T^{*}\right) d \phi\right] e^{-\sigma T^{*}}$. As for the incremental cost, if phase 2 begins at time $T^{*}$ instead of being delayed further, there will be two additional costs in the investment. First, the investment has to be made at $T^{*}$ instead of at a later time, so the amortized investment cost will be increased by $\sigma C\left(T^{*}\right) e^{-\sigma T^{*}}$ because of the time value of money. Second, the potential savings in infrastructure investment can no longer be exploited, which yields a loss of $C^{\prime}\left(T^{*}\right) e^{-\sigma T^{*}}$. Since the company pays for upgrading the infrastructure, the significance of this cost to the regulator is proportional to the weight $\alpha$ that the regulator places on maximizing the company's profit relative to maximizing consumer benefits. The incremental investment is $\alpha\left[\sigma C\left(T^{*}\right)+C^{\prime}\left(T^{*}\right)\right] e^{-\sigma T^{*}}$.

To let the company break-even and to induce it to upgrade the network at $T^{*}$, the regulator has to let the company get a revenue of $\int_{T^{*}}^{+\infty} R\left(T^{*}\right) e^{-\sigma t} d t=\frac{R\left(T^{*}\right)}{\sigma} e^{-\sigma T^{*}}$ per unit of time after phase 2 begins. If $\alpha<1$, asking consumers to pay the company an amount of $\frac{R\left(T^{*}\right)}{\sigma} e^{-\sigma T^{*}}$ decreases the total value of the regulator's objective function (weighted social welfare) by $(1-\alpha) \frac{R\left(T^{*}\right)}{\sigma} e^{-\sigma T^{*}}$. If the upgrade is delayed from time $T^{*}$, this cost can be changed for two reasons: 1) consumers can pay the company later, which yields a marginal savings of $R\left(T^{*}\right) e^{-\sigma T^{*}}$ due to the time-value of money; 2) as the timing changes, the regulator may give the company a different rate increase, which yields a marginal change in the company's revenue of $\frac{R^{\prime}\left(T^{*}\right) e^{-\sigma T^{*}}}{\sigma}$. The necessary condition in Equation 5 can then be derived by letting total incremental benefit equal total incremental cost.

\section{\$3.2 The Impact of Input Parameters}

In this section, we consider how the optimal pricing policy and timing decision are affected by other parameters. Such characterizations are helpful to regulators in making qualitative judgments in deciding prices. Results of our analysis are summarized in Theorem 2 below, followed by some explanations of conclusions. Theorem 2 (see appendix for proof):

Given the optimization problem formulated by Equation 4

a) If the marginal cost of the infrastructure upgrade is smaller, then the optimal time to upgrade the 
infrastructure occurs earlier, i.e. let $T_{1}^{*}, T_{2}^{*}$ be the optimal upgrade time when $C(t)=C_{1}(t)$ and $C(t)=C_{2}(t)$, respectively. If $\sigma C_{1}(t)-C_{1}^{\prime}(t) \leq \sigma C_{2}(t)-C_{2}^{\prime}(t)$ for all $t$, then $T_{1}^{*} \leq T_{2}^{*}$.

b) If the demand for the new feature increases faster, then the optimal time to upgrade the infrastructure occurs earlier, i.e. let $T_{1}^{*}, T_{2}^{*}$ be the optimal upgrade time when $D(\phi, t)=D_{1}(\phi, t)$ and $D(\phi, t)=D_{2}(\phi, t)$, respectively. If $\frac{\partial D_{1}(\phi, t)}{\partial t} \geq \frac{\partial D_{2}(\phi, t)}{\partial t}$ for all $t, \phi$, then $T_{1}^{*} \leq T_{2}^{*}$.

c) If the diffusion for the new feature is faster, then the optimal time to upgrade the infrastructure occurs later, i.e. let $T_{1}^{*}, T_{2}^{*}$ be the optimal upgrade time when $F(\tau)=F_{1}(\tau)$ and $F(\tau)=F_{2}(\tau)$, respectively. If $F_{1}(\tau) \geq F_{2}(\tau)$ for all $\tau, \phi$, then $T_{1}^{*} \geq T_{2}^{*}$.

As a general explanation of Theorem 2, at the optimal upgrade time, $T^{*}$, the incremental benefit of having the new infrastructure begins to surpass the incremental cost. When a change in input parameters leads to a decrease in the incremental cost and/or an increase in incremental benefit, the benefit exceeds the cost earlier, and the optimal upgrade time will also occur earlier.

Theorem 2(a) shows the influence of the investment cost on the optimal timing. One might expect that the optimal upgrade time would occur earlier when the investment cost is lower. The theorem shows this intuition is not always true. As we have discussed in 3.1, the incremental cost of upgrading the new infrastructure includes both the amortized investment cost $\sigma C(t)$ and the lost cost savings $-C^{\prime}(t)$. Only when the sum of these two costs becomes smaller should the optimal timing occur earlier. It is perfectly possible that when $C_{1}(t)>C_{2}(t),-C_{1}^{\prime}(t)$ is smaller than $-C_{2}^{\prime}(t)$, so $\sigma C_{1}(t)-C_{1}^{\prime}(t) \leq \sigma C_{2}(t)-C_{2}^{\prime}(t)$. Consequently, the upgrade should take place later when the investment cost is $C_{2}(t)$ because possible savings in the investment cost from delaying the upgrade is larger.

As for Theorem 2(b), when demand grows faster, the benefit from upgrading the infrastructure increases. This occurs for several reasons. First, for those who want the new feature but can't afford it in phase 1, the value of providing them with the new feature will be greater. Second, the number of adopters of the new feature in phase 1 will increase, which means more customers will experience a reduction in cost when the network is upgraded. Finally, given the same diffusion function, at any given time in phase 1, there will also be more people whose willingness to pay exceeds the price, but have not yet made the purchase. Moving into phase 2 makes the new feature available to them immediately, thus avoiding their potential loss from the delay in subscribing to the new 
feature.

Finally, as shown in Theorem 2(c), when diffusion is slower, there will be more people whose willingness to pay exceeds the phase 1 price, but have not yet made the purchase. Since the phase 2 infrastructure brings the new feature to them immediately, it results in a greater benefit.

It is interesting to note that both faster growth of demand and more rapid diffusion will result in more sales of the new feature in phase 1. However, they lead to an opposite effect on the optimal timing of the upgrade, and therefore on pricing. In other words, sales data alone does not provide sufficient information to make a correct qualitative judgment.

\section{Section 4}

\section{The Static Pricing Policy}

There are a variety proactive pricing approaches that the regulator could adopt to induce the company to upgrade the infrastructure at the socially desirable time. Each approach has its advantages and disadvantages. For example, the regulator could adopt the negligible profit policy under which the company is allowed a revenue increase equal to investment cost plus a small excess profit if it upgrades the infrastructure at the socially desirable time. The company gets no revenue increase at all for other upgrade times. This approach has the advantage of promoting consumer benefit since the company is given very little profit, but will cause the regulator to completely lose control over the timing decision with the slightest underestimate of the investment cost. There are other approaches that offer different tradeoffs, which should be carefully studied. In this paper, we will focus on the discussion of the static pricing policy, which is probably the simplest to implement.

Under the static pricing policy, the regulator allows the company a fixed price increase regardless of when the infrastructure is upgraded. Since the cost of the upgrade does not stay constant, the profit the company gets depends on the upgrade time. The regulator can set the price increase such that the company maximizes profit by upgrading at the socially desirable time. Given demand and cost, this price increase, $R^{*}$, can be determined from the following theorem:

$\underline{\text { Theorem } 3}$ (see Appendix for the proof):

Under the static pricing policy, the company will be induced to upgrade the infrastructure at the optimal time, $T^{*}$, if after the upgrade, the regulator gives the company an additional revenue per unit of time, $R^{*}$, which equals: 


$$
R^{*}=\sigma C\left(T^{*}\right)-C^{\prime}\left(T^{*}\right)
$$

where $T^{*}$ is determined by

$$
\int_{0}^{+\infty} D\left(\phi, T^{*}\right) d \phi-\int_{k_{1}}^{+\infty} X\left(\phi, T^{*}\right) d \phi=\sigma C\left(T^{*}\right)-C^{\prime}\left(T^{*}\right)+(1-\alpha)\left[\frac{C^{\prime \prime}\left(T^{*}\right)}{\sigma}-C^{\prime}\left(T^{*}\right)\right]
$$

Equation 6 shows that the regulator has complete control over the company's timing decision as long as the revenue increase is set correctly. The revenue increase per unit of time needed to induce that timing has two components. The first component $\sigma C\left(T^{*}\right)$ is for recovering the investment cost of upgrading the network, and the second component $-C^{\prime}\left(T^{*}\right)$ is an excess profit the company gets, which equals the cost savings the company gives up by investing at the optimal time $T^{*}$ instead of $T^{*}+d t$. Upgrading at time $T^{*}$ instead of $T^{*}+d t$ reduces the company's revenue by $R d t$, while decreasing the cost by $-C^{\prime}\left(T^{*}\right) d t$, and incurring that cost later, which saves $\sigma C\left(T^{*}\right) d t$. $R$ is constant, and $\sigma C(t)-C^{\prime}(t)$ is always decreasing over time, so there will be only one time, $T^{*}$, when $R^{*}=\sigma C\left(T^{*}\right)$ $C^{\prime}\left(T^{*}\right)$. Consequently, the company will always choose to upgrade at the instant $T^{*}$, when $\sigma C(t)-C^{\prime}(t)$ falls to $R$. As a special case of Theorem 1, Equation 7 specifies the necessary condition for determining the optimal upgrade time under the optimal static pricing policy.

Since the static pricing policy allows the company a revenue increase that exceeds the upgrade cost, yielding a positive profit, a certain amount of wealth will be transferred from consumers to the company. Consequently, maximizing social welfare under the static pricing policy can be beneficial or detrimental to consumers. To prove this point, we first demonstrate there is a potential conflict between maximizing social welfare and maximizing consumer surplus through the example of deploying fiberoptics in the local loop. We then discuss how the optimal timing can be influenced by the regulator's preferences, $\alpha$, towards consumer benefit.

\section{An Example}

Because of the rapid market penetration of home computers and on-line services, an increasing number of customers want to use telephone lines for both data communications and voice service simultaneously. Under the current system, a customer can order a second phone line that connects her computer to the outside world (phase 1). However, the phone company can also upgrade the local loop so all homes in a neighborhood will have the capability to use both phone and data services through a single connection (phase 2). A variety of technical alternatives for upgrading the local loop and their costs have been studied (Reed and Sirbu, 1989; Sirbu et al., 1989). We will use some existing results in our example to demonstrate that the static pricing policy can cause a potential conflict between maximizing social welfare and protecting consumer interests. 
We assume the local loop is going to be upgraded to a triple star network and every household will get 144k bps on their phone line for both voice and data communications. As has been estimated (Sirbu et al., 1989), for a 1,024 home neighborhood over a $10-20$ year time span, the investment cost will drop from $\$ 1934 /$ home to $\$ 947 /$ home, or from approximately $\$ 2,000,000$ to $\$ 1,000,000$ in total. The rate $-C^{\prime}(t)$ at which the cost drops is decreasing over time. We approximate this cost by a continuous function:

$$
C(t)=1,000,000 *\left(e^{-0.5 t}+1\right)
$$

As for consumer demand, we assume that one half of the households (512) are willing to pay $\$ 400$ per year for the new service, and the other half are willing to pay $\$ 50$. The diffusion function is:

$$
F(t)=1-e^{-0.03 t}
$$

Suppose a regulated company is now in phase 1 and has the potential to deploy the fiberoptics in the local loop. We consider the case where the regulator adopts the static pricing policy in Equation 6 to induce her desired time for upgrading the infrastructure. Let the interest rate beyond inflation $\sigma=0.1$. Figures 1 and 2 show social welfare and consumer surplus as functions of deployment time.

\section{[Insert Figure 1]}

\section{[Insert Figure 2]}

As figure 1 shows, the NPV of social welfare first increases and then decreases over the upgrade time. Therefore, to maximize social welfare, the regulator should choose four years from now as the time to deploy the fiberoptics. At this point, the static pricing policy would allow the company to raise the subscription fee for each household by: $\left[\sigma C(4)-C^{\prime}(4)\right] / 1024=\$ 177$ per year, or $\$ 14$ per month, which is a very substantial rate increase. For some users, such an increase almost doubles their monthly subscription fees. This pricing policy allows the company to accumulate about $\$ 1,800,000$ of total revenue, while its investment cost is around $\$ 1,150,000$. In other words, the excess profit the company gets from the upgrade is around $50 \%-60 \%$ of the investment cost.

Figure 2 shows the NPV of consumer surplus, which is increasing over the optimal upgrade time. The dashed line on top of the figure is consumer surplus if the network is never upgraded. Clearly, if the local loop is upgraded at the time that maximizes social welfare, the resulting consumer surplus would be smaller than if the upgrade is never made. Even if the fiberoptics has to be deployed, consumer benefit will be larger if the company deploys at a later date than the time that maximizes social welfare.

This example demonstrates that under certain circumstances, maximizing social welfare can be detrimental to consumer surplus. Therefore, given the same demand and cost parameters, regulators with a different preference 
towards consumer benefits will set price differently. In the introduction to this paper, we formulated the regulator's preference by a weight $\alpha$. When $\alpha$ is smaller, the regulator puts less weight on the company's profit in her objective function. As the following corollary will show, a lower weight on the company's profit (and thus a higher weight on consumer surplus) results in a later upgrade to the infrastructure:

\section{Corollary 1:}

Assume $\left(R_{1}^{*}, T_{1}^{*}\right)$ is the optimal solution to the problem defined in Equation 4 when $\alpha=\alpha_{1}$, and $\left(R_{2}^{*}, T_{2}^{*}\right)$ is the optimal solution when $\alpha=\alpha_{2}$. If $\alpha_{1} \leq \alpha_{2}$ and everything else is kept the same, then:

$$
T_{1}^{*} \geq T_{2}^{*} \text { and } R_{1}^{*} \leq R_{2}^{*}
$$

\section{Section 5}

\section{Conclusions}

In this paper, we consider a regulator's problem of using price controls to induce a desirable time for a profit-maximizing monopoly to upgrade network infrastructure. The upgrade will extend a feature that is currently available to a few subscribers at high cost, and make that feature available to all subscribers as part of basic service.

Existing price regulations, such as rate of return and price cap, cannot give the regulator full control over the timing of the upgrade. Consequently, we investigate proactive pricing policies in which the regulator specifies in advance the additional revenue that a company will be allowed after the upgrade. We derived the necessary condition for determining the optimal timing. We also explored the impact of input parameters. We found that neither a rapid increase in the number of users of the new service nor a lower investment cost will always lead to an earlier optimal upgrade time; other factors must be considered. Furthermore, the optimal upgrade time can occur later in cases when diffusion is faster, or in cases when the investment cost falls rapidly.

There are a variety of proactive pricing approaches that a regulator could use for inducing the optimal timing. In this paper, we focus our discussion on the static pricing policy. We demonstrate that because this pricing policy gives an excess profit to the regulated company, it creates a conflict between social welfare and consumer surplus. We also discuss the influence of the regulator's preference for maximizing social welfare versus maximizing consumer surplus on the optimal timing, and demonstrate that when the regulator is more focused on the consumer benefits, the optimal timing should be delayed.

Of course, there are other possible proactive pricing schemes. Since the disadvantages of the static pricing policy come primarily from the excess profit that all effective proactive schemes will have, the tradeoffs described 
above are illustrations of the problems to be addressed. Further work is needed to explore other proactive pricing policies that balance the conflicting objectives, such as social welfare maximization, consumer surplus maximization, simplicity, and robustness in the face of cost and demand uncertainty. 


\section{Appendix}

\section{Proof of Theorem 1:}

Take the first-order derivative of $\Pi(T)$ (see Equation 4) with respect to $T$ :

$$
\begin{aligned}
\frac{d \Pi}{d T}=\int_{k_{1}}^{+\infty} & X(\phi, T) d \phi e^{-\sigma T}-\int_{0}^{+\infty} D(\phi, T) d \phi e^{-\sigma T} \\
& +(1-\alpha)\left[\frac{R^{\prime}(T)}{\sigma}-R(T)\right] e^{-\sigma T}+\alpha\left[\sigma C(T)-C^{\prime}(T)\right] e^{-\sigma T}
\end{aligned}
$$

Since at the optimal timing $T^{*}, \frac{d \Pi}{d T}=0$

$$
\int_{0}^{+\infty} D\left(\phi, T^{*}\right) d \phi-\int_{k_{1}}^{+\infty} X\left(\phi, T^{*}\right) d \phi=\alpha\left[\sigma C\left(T^{*}\right)-C^{\prime}\left(T^{*}\right)\right]+(1-\alpha)\left[R\left(T^{*}\right)-\frac{R^{\prime}\left(T^{*}\right)}{\sigma}\right] \quad \text { Q. E. D. }
$$

\section{Proof of Theorem 2 and Corollary 1:}

Theorem 2(a), (b),(c), and Corollary 1 can be proven by the same approach. In the following, we demonstrate that approach by proving Theorem 2(a):

Proof Let $T_{1}^{*}$ be the optimal timing and $\Pi_{1}\left(T_{1}^{*}\right)$ be the resulting weighted social welfare when $C(t)=C_{1}(t)$. Let $T_{2}^{*}$ be the optimal timing and $\Pi_{2}\left(T_{2}^{*}\right)$ be the resulting weighted social welfare when $C(t)=C_{2}(t)$.

By assumption, $\Pi_{1}\left(T_{1}^{*}\right) \geq \Pi_{1}\left(T_{2}^{*}\right)$ and $\Pi_{2}\left(T_{2}^{*}\right) \geq \Pi_{2}\left(T_{1}^{*}\right)$, which lead to:

$$
\begin{aligned}
& \int_{T_{2}^{*}}^{T_{1}^{*}} \int_{k_{1}}^{*} X(\phi, t) d \phi e^{-\sigma t} d t-\int_{T_{2}^{*}}^{T_{1}^{*}} \int_{0}^{\infty} D(\phi, t) d \phi e^{-\sigma t} d t \\
& -(1-\alpha)\left[R_{1}\left(T_{1}^{*}\right) e^{-\sigma T_{1}^{*}}-R_{1}\left(T_{2}^{*}\right) e^{-\sigma T_{2}^{*}}\right]-\alpha\left[C_{1}\left(T_{1}^{*}\right) e^{-\sigma T_{1}^{*}}-C_{1}\left(T_{2}^{*}\right) e^{-\sigma T_{2}^{*}}\right] \geq 0
\end{aligned}
$$

and

$$
\begin{aligned}
& \int_{T_{2}^{*}}^{T_{1}^{*}+\infty} X(\phi, t) d \phi e^{-\sigma t} d t-\int_{T_{2}^{*}}^{T_{1}^{*}} \int_{0}^{\infty} D(\phi, t) d \phi e^{-\sigma t} d t \\
& -(1-\alpha)\left[R_{2}\left(T_{1}^{*}\right) e^{-\sigma T_{1}^{*}}-R_{2}\left(T_{2}^{*}\right) e^{-\sigma T_{2}^{*}}\right]-\alpha\left[C_{2}\left(T_{1}^{*}\right) e^{-\sigma T_{1}^{*}}-C_{2}\left(T_{2}^{*}\right) e^{-\sigma T_{2}^{*}}\right] \leq 0
\end{aligned}
$$

If the regulator adopts the same pricing policy, the excess profit to induce the company to upgrade the infrastructure at time $T_{1}^{*}$ and time $T_{2}^{*}, \pi\left(T_{1}^{*}\right)$ and $\pi\left(T_{2}^{*}\right)$, should be the same for both the case when $C(t)=C_{1}(t)$ and the case when $C(t)=C_{2}(t)$, i.e.:

$$
R_{1}\left(T_{1}^{*}\right)=\sigma C_{1}\left(T_{1}^{*}\right)+\pi\left(T_{1}^{*}\right), \quad R_{2}\left(T_{1}^{*}\right)=\sigma C_{2}\left(T_{1}^{*}\right)+\pi\left(T_{1}^{*}\right)
$$


and

$$
R_{1}\left(T_{2}^{*}\right)=\sigma C_{1}\left(T_{2}^{*}\right)+\pi\left(T_{2}^{*}\right)
$$$$
R_{2}\left(T_{2}^{*}\right)=\sigma C_{2}\left(T_{2}^{*}\right)+\pi\left(T_{2}^{*}\right)
$$

Therefore

$$
\begin{aligned}
& \int_{T_{2}^{*}}^{T_{1}^{*}+\infty} X(\phi, t) d \phi e^{-\sigma t} d t-\int_{T_{2}^{*}}^{T_{1}^{*}} \int_{0}^{+\infty} D(\phi, t) d \phi e^{-\sigma t} d t \\
& \quad-(1-\alpha)\left[\pi\left(T_{1}^{*}\right) e^{-\sigma T_{1}^{*}}-\pi\left(T_{2}^{*}\right) e^{-\sigma d_{2}^{*}}\right]-\left[C_{1}\left(T_{1}^{*}\right) e^{-\sigma T_{1}^{*}}-C_{1}\left(T_{2}^{*}\right) e^{-\sigma T_{2}^{*}}\right] \geq 0 \\
& \text { and } \quad \int_{T_{2}^{*}} \int_{k_{1}} X(\phi, t) d \phi e^{-\sigma t} d t-\int_{T_{2}^{*}}^{T_{1}^{*}+\infty} D(\phi, t) d \phi e^{-\sigma t} d t \\
& \quad-(1-\alpha)\left[\pi\left(T_{1}^{*}\right) e^{-\sigma T_{1}^{*}}-\pi\left(T_{2}^{*}\right) e^{-\sigma \pi_{2}^{*}}\right]-\left[C_{2}\left(T_{1}^{*}\right) e^{-\sigma T_{1}^{*}}-C_{2}\left(T_{2}^{*}\right) e^{-\sigma T_{2}^{*}}\right] \leq 0
\end{aligned}
$$

which lead to:

$\left[C_{2}\left(T_{1}^{*}\right) e^{-\sigma T_{1}^{*}}-C_{2}\left(T_{2}^{*}\right) e^{-\sigma T_{2}^{*}}\right] \geq\left[C_{1}\left(T_{1}^{*}\right) e^{-\sigma T_{1}^{*}}-C_{1}\left(T_{2}^{*}\right) e^{-\sigma T_{2}^{*}}\right]$

Since

$$
\begin{aligned}
& \int_{T_{2}^{*}}^{T_{1}^{*}}\left\{\left[\sigma C_{1}(t)-C_{1}^{\prime}(t)\right]-\left[\sigma C_{2}(t)-C_{2}^{\prime}(t)\right]\right\} e^{-\sigma t} d t \\
& \quad=\left[C_{2}\left(T_{1}^{*}\right) e^{-\sigma T_{1}^{*}}-C_{2}\left(T_{2}^{*}\right) e^{-\sigma T_{2}^{*}}\right]-\left[C_{1}\left(T_{1}^{*}\right) e^{-\sigma T_{1}^{*}}-C_{1}\left(T_{2}^{*}\right) e^{-\sigma T_{2}^{*}}\right] \geq 0
\end{aligned}
$$

and $\quad\left[\sigma C_{1}(t)-C_{1}^{\prime}(t)\right] \leq\left[\sigma C_{2}(t)-C_{2}^{\prime}(t)\right]$,

$$
T_{1}^{*} \leq T_{2}^{*}
$$

Q. E. D.

\section{Proof of Theorem 3:}

a) The company's profit function is:

$$
\begin{aligned}
& \Gamma(T)=\int_{T}^{+\infty} R e^{-\sigma t} d t-C(T) e^{-\sigma T} \\
& \frac{d \Gamma}{d T}=0 \text { and } \frac{d^{2} \Gamma}{d T^{2}} \leq 0 \text { at } T=T^{*} \text { iff } R=\sigma C\left(T^{*}\right)-C^{\prime}\left(T^{*}\right)
\end{aligned}
$$

Therefore, the company's profit is maximized at when $T=T^{*}$, so the company will be induced to upgrade the infrastructure at that time, iff $R=\sigma C\left(T^{*}\right)-C^{\prime}\left(T^{*}\right)$

b) Plug $R=\sigma C\left(T^{*}\right)-C^{\prime}\left(T^{*}\right)$ into the necessary condition of Equation 5 .

Q. E. D. 


\section{References}

Bass, F.M., 1969, "A New Product Growth Model for Consumer Durables," Management Science, vol. 15 (January), 215-227.

Johnson, L., 1989, "Price Caps in Telecommunications Regulatory Reform," Rand Note, N-2894-MF/RC, (January).

Mitchell, B.M. and I. Vogelsang, 1991, Telecommunications Pricing, (Cambridge University Press).

Noll, R.G., 1989, “Telecommunications Regulation in the 1990s," New Directions in Telecommunications Policy, edited by Paula R. Newberg, (Duke University Press), 11 - 48.

Reed, D.P. and M.A. Sirbu, 1989, “An Optimal Investment Strategy Model for Fiber to the Home," Journal of Lightwave Technology, vol. 7, no. 11 (November), 1868-1875.

Sirbu, M.A., D. P. Reed, and F.E. Ferrante, 1989, “An Engineering and Policy Analysis of Fiber Introduction into the Residential Subscriber Loop,” Journal of Lightwave Technology, vol. 7, no. 11 (November), 1876-1884.

Wrobel, L.A., 1990, Disaster Recovery Planning for Telecommunications, (Artech House).

Wu, T.-H., 1992, Fiber Network Service Survivability, (Artech House). 
Table 1

Variables Used in the Mathematical Model

\begin{tabular}{|c|c|c|}
\hline Symbols & Definitions & Properties and Constraints \\
\hline & Decision Variables & \\
\hline$p$ & $\begin{array}{l}\text { Subscription fee per unit of time for using } \\
\text { the new feature in phase } 1\end{array}$ & $p=k_{1}$ \\
\hline$r(t, T)$ & $\begin{array}{l}\text { Additional revenue per unit of time the } \\
\text { company gets after it upgrades the } \\
\text { infrastructure at time } t \text {, assuming the } \\
\text { regulator expects the upgrade takes place at } \\
T \text {. }\end{array}$ & $R(T)=r(T, T)$ \\
\hline \multirow[t]{3}{*}{$T$} & Regulator desired time to upgrade the & $0 \leq T \leq+\infty$ \\
\hline & infrastructure & if $T=+\infty$, then Phase 1 lasts forever. \\
\hline & Demand & \\
\hline$D(\phi, t)$ & $\begin{array}{l}\text { Number of subscribers who are willing to } \\
\text { pay at least } \phi \text {, at time } t \text {, for the new } \\
\text { feature. }\end{array}$ & $D(\phi,-\infty)=0, \frac{\partial D(\phi, t)}{\partial \phi} \leq 0, \frac{\partial D(\phi, t)}{\partial t} \geq 0$ \\
\hline$F(\tau)$ & Diffusion function. & $F(\tau) \geq 0, \frac{d F(\tau)}{d \tau} \geq 0, F(+\infty)=1$ \\
\hline \multirow[t]{2}{*}{$X(\phi, t)$} & $\begin{array}{l}\text { Number of subscribers who would } \\
\text { subscribe to the new feature at time } t \text { in } \\
\text { phase } 1 \text {, if price is } \phi \text {. }\end{array}$ & $X(\phi, t)=\int_{-\infty}^{t} \frac{\partial D(\phi, \mu)}{\partial \mu} F(t-\mu) d \mu$ \\
\hline & Cost & \\
\hline$C(T)$ & $\begin{array}{l}\text { Fixed cost of upgrading the infrastructure } \\
\text { at time } T \text {. }\end{array}$ & $\frac{d C(T)}{d T} \leq 0, \frac{d^{2} C(T)}{d T^{2}} \geq 0, C(T) \geq 0$ \\
\hline$k_{1}$ & $\begin{array}{l}\text { Cost per customer per unit of time of } \\
\text { providing the new feature in phase } 1 .\end{array}$ & \\
\hline$\sigma$ & Discount rate. & $\sigma>0$ \\
\hline
\end{tabular}




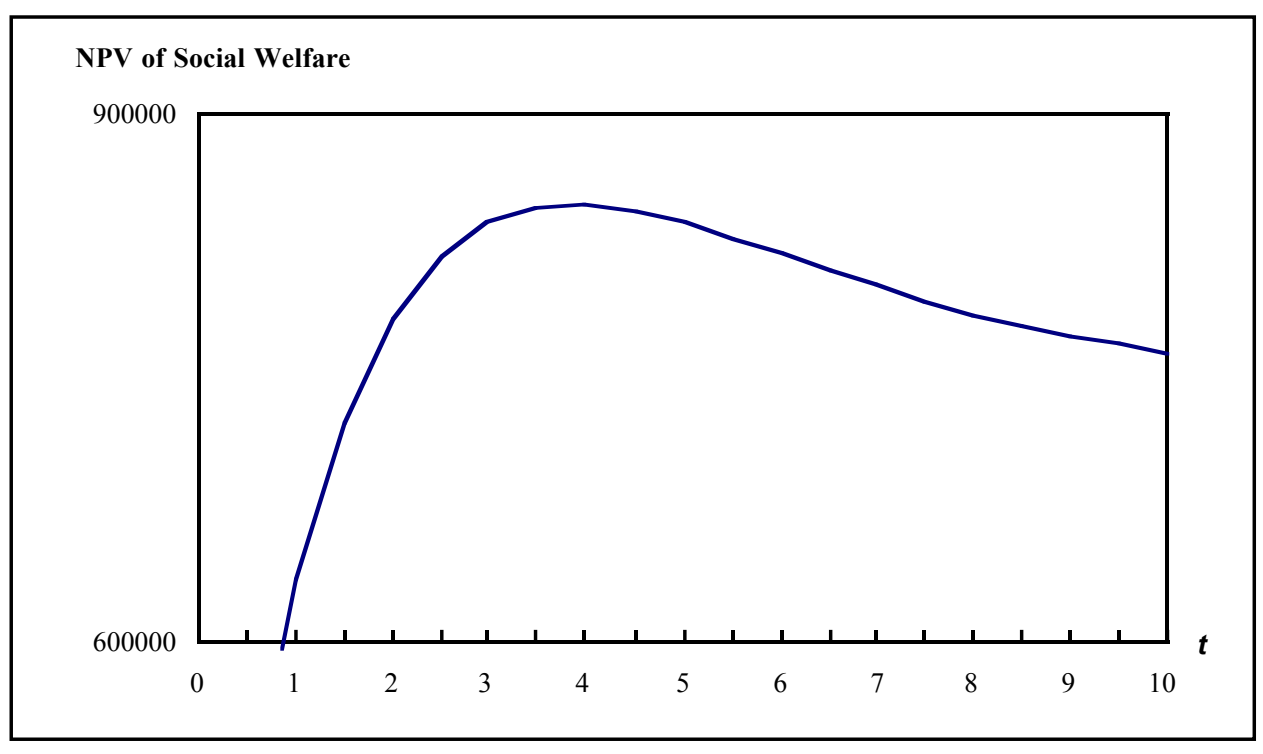

Figure 1

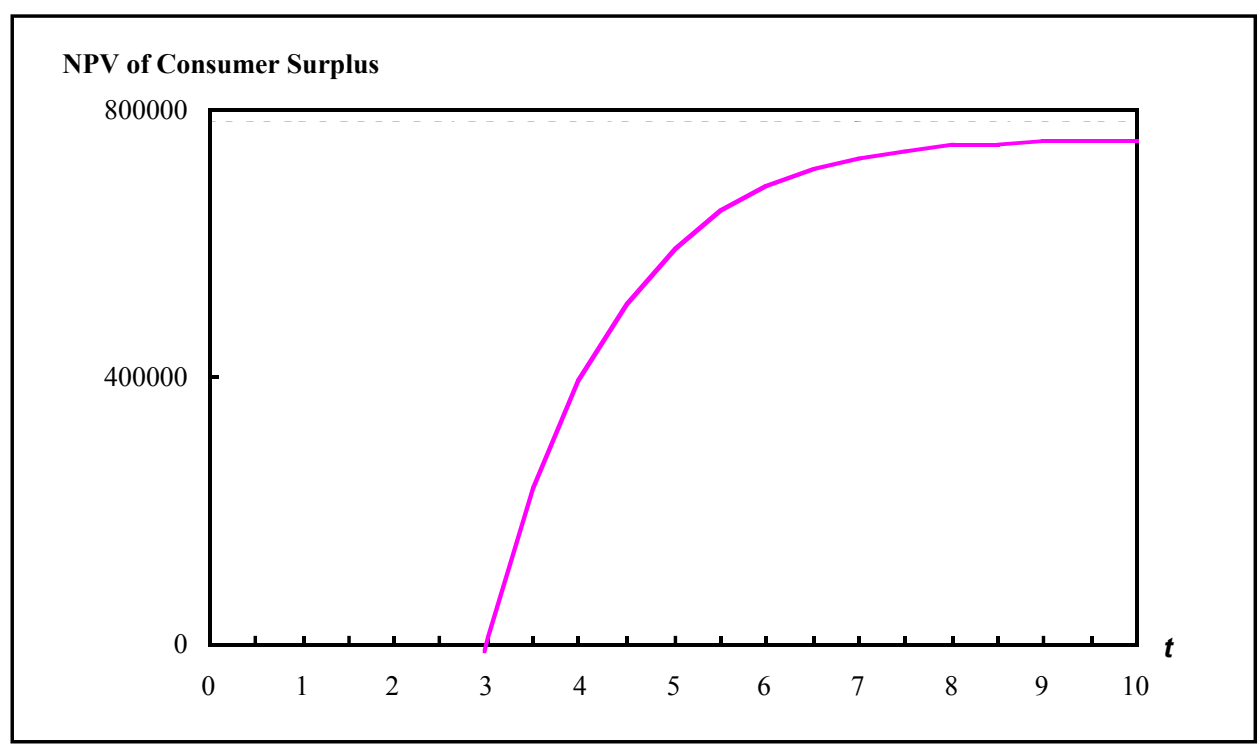

Figure 2 